\title{
OBTENÇÃO E VALIDAÇÃO DE PARÂMETROS DA MOF ZIF-8 PARA SIMULAÇÃO ATOMÍSTICA POR DINÂMICA MOLECULAR
}

\author{
Raquel Eufrasio Medeiros Lins ${ }^{1}$ Frederico José de Santana Pontes ${ }^{1}$, Thereza Amélia Soares da Silva ${ }^{1}$ \\ ${ }^{1}$ BioMat - Biomaterials Modeling Group, UFPE. Departamento de Química Fundamental/CCEN \\ *quelmlins@gmail.com
}

\begin{abstract}
INTRODUÇÃO
Neste trabalho utilizamos simulações computacionais de dinâmica molecular (MD) para investigar propriedades microscópicas de Metal Organic Frameworks (MOFs) em meio aquoso. MOFs formam uma classe materiais porosos com forte interação entre metal e ligantes e que podem assumir diferentes propriedades físicas e quí micas, incluindo diferentes tamanhos e estrutura dos poros, acidez, propriedades óticas, magnéticas, etc. Nosso principal interesse é estudar transições estruturais de MOFs sob efeito de diferentes condições físico-químicas com o intuito de utilizar essas transformações estruturais para a liberação/encapsulação de drogas.
\end{abstract}

Uma das classes de MOFs mais comuns e largamente estudadas são as zeólitas contendo anéis imidazois em sua estrutura, conhecidas como ZIF (zeolitic imidazolate framework). As ZIFs são conhecidas pela sua grande porosidade e alta estabilidade térmica (até $550{ }^{\circ} \mathrm{C}$ ) e química frente a condições alcalinas e solventes orgânicos [PARK, 2006]. Essas classes de moléculas apresentam transições de fases estruturais em diferentes condições de temperatura e solvente devido a sua flexibilidade estrutural [ZHAO, 2014]. Essa caracterí stica estrutural torna essa classe de material como um promissor carreador e liberador de fármacos.

Um dos melhores representantes dessa classe é a ZIF-8 que já vem sendo largamente estudada por técnicas experimentais e teóricas como carreadoras de drogas [VASCONCELOS, 2014, SUN, 2012] e liberador de drogas em diferentes condições químicas. Apesar de todas essas características fascinantes, detalhes atomísticos dos processos de transformação estrutural, carreamento e liberação de fármacos continuam em aberto necessitando de investigações mais profundas. Nesse cenário, a dinâmica molecular torna-se uma ferramenta indispensável para fornecer detalhes e fazer predições sobre o comportamento molecular desses sistemas.

Nesse trabalho comparamos parâmetros para simulação computacional por MD da ZIF-8 disponíveis na literatura [ZHENG, 2012, ZHONGQIAO, 2012] com parâmetros desenvolvidos por nós utilizando o método GAFF (General AMBER Force Field) disponível no pacote Antechamber [WANG, 2006, WANG, 2004].

\section{MATERIAIS E MÉTODOS}

A escolha de parâmetros adequados para a simulação dos sistemas envolveu uma busca bibliográfica na literatura especializada, além do estabelecimento de um protocolo sistemático para a obtenção de novos parâmetros de simulação. Enquanto a parte orgânica pode ser descrita pelos principais campos de força existentes, é necessá rio determinar a interação entre esses ligantes e os centros metá licos. Devido à maior disponibilidade de parâmetros para íons metá licos, adotou-se o campo de força AMBER [WANG, 2004] para a construção das topologias. Escolheu-se então uma MOF extensivamente descrita na literatura (ZIF-8) com diferentes conjuntos de parâmetros [ZHENG, 2012, ZHONGQIAO, 2012] além da parametrização do resíduo através das ferramentas descritas no pacote AmberTools [WANG, 2004].

Os parâmetros foram testados em dois conjuntos de simulações computacionais. Primeiramente simulamos células unitárias da ZIF8 de tamanho $1 \times 1 \times 1$ em água. Em seguida, uma análise mais minuciosa dos parâmetros foi feita através da simulação de uma ú nica molécula de imidazol em água.

As simulações ocorreram com número de partículas, temperatura $(298 \mathrm{~K})$ e pressão (1 bar) constantes, caracterizando um ensemble NpT. O passo de integração foi de 2 fs. Anterior à simulação computacional o sistema foi submetido a uma minimização de energia utilizando o algoritmo de steepest descent. A construção das caixas, simulações computacionais e posteriores análises foram realizadas com o programa GROMACS 4.5.x [PRONK, 2013].

\section{RESULTADOS E DISCUSSÃO}

As simulações envolvendo os parâmetros disponíveis na literatura apresentaram problemas estruturais.

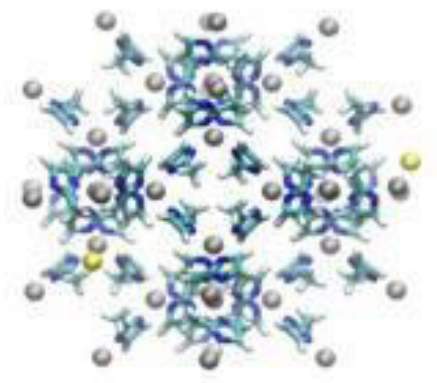

(a)

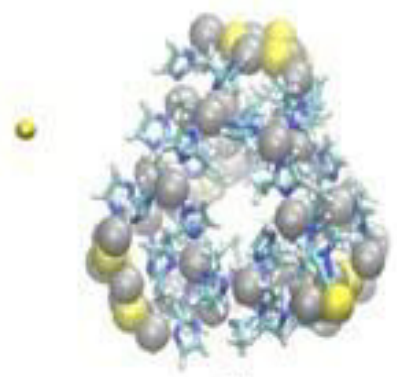

(b)
Figura 1. Estrutura inicial da ZIF-8 no começo da simulação (a) e no final da simulação (b). Átomos de zinco e cloro representados pelas cores cinza e amarelo, respectivamente. As moléculas de água não foram representadas por questão de clareza.

Analisando intimamente a estrutura, observamos grandes distorçõ es estruturais na parte orgânica da ZIF. Os anéis do ligante orgâ nico não se mantiveram planares ao longo da simulação revelando um problema com os parâmetros disponíveis e necessitando uma análise local do problema. Realizamos então simulações apenas da parte orgânica do sistema em água para caracterizar suas propriedades estruturais e dinâmicas. 
Nessa etapa analisamos o deslocamento médio da estrutura durante a simulação e os diedros impróprios responsáveis pela planaridade do sistema.

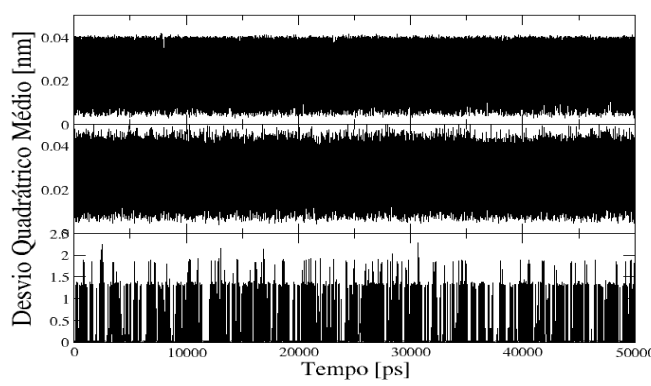

Figura 2. Desvio quadrático médio do ligante orgânico da ZIF-8. Valores obtidos com parâ metros do GAFF (topo), referência 5 (meio) e referência 6 (base).

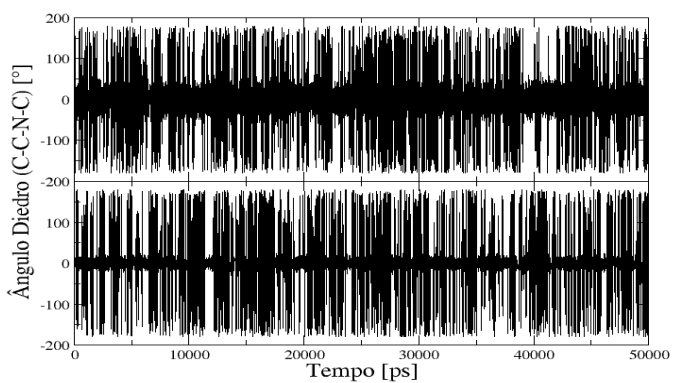

Figura 3. Comportamento do ângulo diedro envolvendo os átomos de C-C-N-C do ligante orgânico da ZIF-8 ao longo do tempo. Valores obtidos com referência 5 (topo) e referê ncia 6 (base).

As distorções obtidas nas simulações envolvendo a célula unitária da ZIF-8 podem ser em parte explicadas pela forma em que 0 arquivo binário de simulação foi construído. Utilizou-se uma ferramenta disponível no programa GROMACS chamada g_x2top que não codifica direito os diedros impróprios e por isso a estrutura sofre uma grande distorção no ligante orgânico da MOF.

No restante do trabalho, utilizamos outra ferramenta do programa GROMACS (pdb2gmx) para manipular os arquivos de parâmetros. Realizamos simulacões da parte orgânica da ZIF-8 numa caixa de á guas e íons suficientes para neutralizar o sistema. Nesse novo conjunto de simulações os diedros impróprios foram corretamente codificados no arquivo binário de simulação, mas encontramos diferentes descrições entre os campos de força nas análises estruturais.

As análises apresentaram que em termos energéticos, de hidrataçã o e ligações de hidrogênio os três conjuntos de parâmetros tiveram desempenho equivalente. A distinção entre eles pode ser observada apenas nas análises estruturais.

A análise do desvio quadrático médio dos três conjuntos de parâ metros (Figura 2) indica que apesar de bastante baixo, o valor da propriedade oscila bastante e para um valor bastante grande quando utilizamos o conjunto de parâmetros provenientes da ref. 6. Outro ponto importante que observamos, foram os ângulos diedros envolvendo os átomos de C - C - N - C do anel imidazol, um forte indicativo da planaridade do sistema. Esse ângulo que deveria ter o valor de zero grau e ser um indicador da planaridade do anel apresenta grandes variações em seu valor para os conjuntos de parâmetros publicados nas referências 5 e 6 .

Apenas o conjunto de parâmetros obtidos com a metodologia GAFF mostrou-se capaz de gerar uma estrutura do ligante orgânico estruturalmente estável indicando ser a melhor escolha para consolidarmos uma metodologia para a simulação por dinâmica molecular de MOFs.

\section{CONCLUSÕES}

Com o final das simulações teste, foi possível concluir que a estrutura que apresentou melhores parâmetros foram as que foram calculadas pelo GAFF obtido com a ferramenta Antechamber. Sua estrutura foi a que melhor representou a estrutura do imidazol, sofrendo apenas pequenas torções em seus ângulos e diedros. E também a que menos sofreu mudanças com relação a sua estrutura inicial. Como perspectiva, encontra-se obter novos parâmetros de interação entre a parte orgânica e o átomo metálico $\left(\mathrm{Zn}^{2+}\right)$ através de metodologia já estabelecida na literatura e prosseguir com as simulações computacionais.

\section{REFERÊNCIAS}

PARK, K. S. et al. Exceptional chemical and thermal stability of zeolitic imidazolate frameworks. Proceedings of The National Academy Of Sciences, v. 103, n. 27, p.10186-10191, 23 jun. 2006. ZHAO, Pu et al. Phase Transitions in Zeolitic Imidazolate Framework 7: The Importance of Framework Flexibility and GuestInduced Instability. Chemistry Of Materials, v. 26, n. 5, p.17671769, 21 fev. 2014.

VASCONCELOS, I. B. et al.Cytotoxicity and Slow Release of the Anti-cancer Drug Doxorubicin from ZIF-8. RSC Advances, v. 2, p. 9437, 2012.

SUN, Chun-yi et al. Zeolitic imidazolate framework-8 as efficient $\mathrm{pH}$-sensitive drug delivery vehicle. Dalton Transactions. Royal Society of Chemistry (RSC),v. 41, n. 23, p.6906-6909, 2012.

ZHENG, Bin et al. Force Field for Molecular Dynamics Computations in Flexible ZIF-8 Framework. The Journal Of Physical Chemistry, v. 116, n. 1, p.933-938, 4 jan. 2012.

ZHONGQIAO et al. Development of a Force Field for Zeolitic Imidazolate Framework-8 with Structural Flexibility. Journal of Physical Chemistry, v. 136, n.24, p. 244703, 28 jun. 2012.

WANG, Junmei et al. Automatic atom type and bond type perception in molecular mechanical calculations. Journal of Molecular Graphics And Modelling, v. 25, n. 2, p.247-260, out. 2006.

WANG, Junmei et al. Development and testing of a general amber force field. Journal of Computational Chemistry, v. 25, n. 9 p.1157-1174, 2004. Wiley-Blackwell.

PRONK, Sander et al. GROMACS 4.5: a high-throughput and highly parallel open source molecular simulation toolkit. Bioinformatics, v. 29, n. 7, p.845-854, 13 fev. 2013. 